\title{
Éditorial
}

\section{Le « normal » existe-t-il en orthodontie ? Petit commentaire subversif et irrévérencieux à l'usage des jeunes générations}

Q uand j'entends le mot normal, je consulte mes référents lexicaux. Larousse me dit : qui est conforme au caractère le plus fréquent (c'est-à-dire $50 \%+1$ ?). Pour norme, il dit : état habituel conforme à la majorité des cas (c'est-à-dire $50 \%+1$ ?). Quant à Robert, il me propose pour normal : conforme à une moyenne considérée comme une norme. Pour norme, il déclare : état habituel conforme à la règle établie. Le serpent se mord la queue...

Les questions essentielles pour l'orthodontiste restent hélas sans réponse.

Qui décide du normal ? C'est quoi exactement « la majorité des cas »? Qui établit la règle, de quelle autorité ? Est-il est souhaitable d'être normal ou dans la norme?

Quel bénéfice mesurable m'apporte le fait d'être normal ou dans la norme? À quels malheurs suis-je exposé si je ne suis pas dans la norme ? Ceux qui ne sont pas dans la norme doivent-ils être qualifiés " d'anormaux »? Devrait-on plutôt considérer qu'« anormal » signifierait « moins habituel » ou « moins fréquent » ou « différent »?

Incertitude, indétermination, imprécision, flou, vague, voilà quelques caractéristiques du mot « normal » appliqué à l'orthodontie.

Normal ou norme sont pourtant deux mots omniprésents dans la conversation orthodontique. Les possibles approches ou définitions du normal en santé peuvent être les suivantes (Grenier, ed. Masson): -1 critère statistique $(\mathrm{M}+/$ - ET). Proposition qu'on ne peut biologiquement approuver car il s'agit d'un concept théorique conventiondépendant. On retiendra pourtant de cette approche que la règle en biologie est la diversité ou variabilité et non pas l'uniformité et qu'une grande partie de nos corrections ne font que traiter de variations du normal statistique ; -2 critère prédictif. En fonction du risque de pathologie, les limites du normal sont celles au delà desquelles la probabilité d'une maladie devient suffisamment élevée ; -3 critère pragmatique : les limites du normal sont celles au-delà desquelles les risques de morbidité s'accroissent suffisamment pour justifier une intervention médicale. 
Mais la malocclusion est-elle réellement une maladie, c'est-à-dire un processus morbide qui aboutit à une dégradation de l'Individu ? La HAS elle-même précise qu'il existe une multitude d'occlusions possibles, non pathogènes et efficaces. Dans quelle mesure la malocclusion constitue-t-elle un risque pour la santé ? Quelle est la menace réelle de la malocclusion pour parodonte, les ATM, le risque de caries ? Certes, des risques peuvent exister, mais le fardeau de la preuve fait souvent défaut et le débat est loin d'être clos. Heureusement, dans un autre esprit, l'OMS nous précise que la santé est un état de complet bien-être physique, mental, social et pas seulement l'absence de maladie ou d'infirmité. Nul ne contestera que dans une société qui pratique le culte du beau, la qualité d'un sourire puisse être un facteur de réussite, d'intégration sociale, d'estime de soi, de santé globale. La définition du normal est aujourd'hui devenue le fait d'éléments sociaux. Ce fait social reste l'aspect fort de la justification de notre activité. Il est ainsi aujourd'hui impossible de faire abstraction de ce fait de société. Se soumettre à la norme revient donc à être dans le devoir et l'orthodontiste est bel et bien dans le devoir : j'accepte les diktats du moment. Je remercie donc le fait social du jour de la chance qu'il m'offre d'exercer mon métier.

Reste que la mesure exacte du handicap occlusal associé à la malocclusion est difficile à chiffrer car il est le plus souvent qualitatif. Quand je pose la question à un amphithéâtre d'étudiants « y a-t-il ici quelqu'un d'anormal ? ", je ne vois en général aucune main se lever. J'en conclus (hâtivement ?) que chacun aurait volontiers tendance à admettre et ressentir son propre modèle comme une référence parfaitement acceptable du normal et que donc le normal varie avec les exigences personnelles et non les moyennes statistiques (l'anormal serait sans doute plutôt l'autre...).

Est-il alors éthique que j'explique à mon patient à quel point il n'est pas dans la norme et combien il lui serait profitable que je l'y remette par un traitement orthodontique approprié. Commençons plutôt notre dialogue en lui posant simplement la question " qu'attendez-vous de moi ? " puis continuons en essayant de répondre à sa demande. Ne jetons pas trop hâtivement notre patient dans cette vaste enveloppe où l'on exile tous ceux qui ne sont pas inscrits dans la norme, cette grande boîte dont nous définissons souvent nous-mêmes les limites. Inhabituel ne signifie pas nécessairement pathologique et normal occlusal ne veut pas nécessairement dire idéal théorique.

Maudite soit la norme.

William Bacon

Professeur, Responsable de la Structure Interne d'Orthopédie Dento-Faciale des Hôpitaux Universitaire de Strasbourg

\author{
"L'association de la Revue d'Orthopédie Dento-Faciale \\ et son comité de rédaction \\ vous présentent leurs meilleurs vœux pour 2014 »
}

